\title{
Dietmar Willoweit
}

\section{Rechtsbegründung und Rechtsbegriff}

\author{
Ein Nachwort
}

I.

Ein Kolloquium über Probleme der Rechtsbegründung kann sich nicht der Öffentlichkeit präsentieren, ohne die spezifischen Legitimationsprobleme unserer jüngeren Geschichte und Rechtsgeschichte anzusprechen. In welchem Zusammenhang steht die Frage nach der Begründung des Rechts mit der Frage nach den Grenzen des Rechts und der Erfahrung von Unrecht? Ist jedes staatliche Handeln der Begründung in der Dimension des Rechts zugänglich? Darauf gibt es zunächst die logische Antwort: Bedingung der Möglichkeit einer Begründung des Rechts ist der Begriff des Rechts. Nun ist es kein Geheimnis, daß mit dem Stichwort "Rechtsbegriff“" ein Problem angesprochen ist, das jedenfalls in jüngerer Zeit als kaum lösbar galt und daher von manchem als letztlich unlösbar beiseite gelegt worden ist. Sich dabei zu beruhigen, würde in unserem Zusammenhang bedeuten: Recht ist, was sich selbst so nennt, so daß sich die Begründungsfrage in der Tat auf alle einschlägigen historischen Phänomene erstrecken würde, auf jedes staatliche Handeln, jeden hoheitlichen Befehl. Für diesen Standpunkt kann man sogar ins Feld führen, daß sich die Frage nach der Begründung, der Rechtfertigung, der Legitimation hoheitlichen Handelns in der Tat so umfassend stellt, wie die Staatsgewalt tatsächlich tätig wird. Das Thema "Rechtsbegründung und Rechtsbegriff“ würde sich also als gegenstandslos erweisen, weil die Begründungsfrage an den Staat zu richten wäre, dessen Handeln weit über den Raum des Rechts hinausreicht.

Diese Überlegungen helfen allerdings dann nicht weiter, wenn - wie hier - das Tagungskonzept der Unterstützung eines umfassenderen rechtsgeschichtlichen Forschungsvorhabens dient, das unter dem Arbeitsthema "Recht und Gesellschaft" davon ausgeht, daß Recht ein wesentliches Element jeder funktionsfähigen Gesellschaftsformation ist. Eine Fragestellung dieser Art kann auf die Klärung ihres Vorbegriffs von Recht nicht verzichten. Andernfalls würde die Auswahl der zu berücksichtigenden historischen Fakten zufällig und methodisch unkontrolliert erfolgen. Einfacher ausgedrückt: Wer Rechtsgeschichte betreibt, 
kann dies ohne einen Begriff von Recht nicht tun. Dabei mag es in der Regel genügen, wenn das Vorverständnis für den Forschungsgegenstand ${ }_{n}$ Recht “ ungefähr aus den Erfahrungen mit der modernen Rechtsordnung abgeleitet wird. Wenn man aber versucht, Recht nicht von vorneherein als Äußerung hoheitlicher, selbst erst in historischer Zeit entstehender Mächte zu verstehen, sondern der Entstehung von Recht aus der Gesellschaft heraus nachzugehen, dann allerdings ist ein präziserer Rechtsbegriff unverzichtbar, weil man wissen muß, was man sucht. Meine These ist nun, daß es in der Tat möglich ist, einen solchen Rechtsbegriff mit philosophischer Nachhilfe in der Geschichte dingfest zu machen, so daß am Ende doch ein Spannungsverhältnis zwischen „Rechtsbegründung" und "Rechtsbegriff" entsteht.

Der philosophische Kronzeuge, auf den ich mich berufe, ist Rudolf Stammler. In seiner Abhandlung ${ }_{n}$ Recht und Willkür ${ }^{" 1}$ hat er den Rechtsbegriff mit logischen Überlegungen so eingegrenzt, daß er geeignet ist, grundlegende Hypothesen der Rechtsgeschichte zu bestätigen. Ich versuche, den Gedankengang Stammlers hier in aller Kürze nachzuzeichnen und seine Tauglichkeit für die Zwecke rechtsgeschichtlicher Forschung wahrscheinlich zu machen, um anschließend einen Blick auf die im 20. Jahrhundert zutage getretenen Probleme zu werfen. Auch dazu bietet Stammler, obwohl er seinen Text im wesentlichen schon im Jahre 1895 formulierte, anregende Gedanken².

Stammler fragt zunächst nach dem unterscheidenden Kriterium zwischen Rechtsnormen und Konventionalregeln. Er findet es in dem Geltungsanspruch der Rechtsnorm, die ihrem immanenten Sinn gemäß Beachtung fordert, ohne Rücksicht darauf, ob die Adressaten der gebotenen Regelung zustimmen oder nicht. Konventionalregeln dagegen erheben einen solchen Anspruch nicht, jedenfalls nicht im Sinne eines Zwangsgebotes. Ihre Befolgung beruht im Prinzip auf dem Einverständnis der solchen Regeln unterworfenen Personen. Wenn aber die Geltung einer Rechtsnorm keinesfalls auf die Anerkennung durch die Normadressaten zurückgeführt werden kann, dann stellt sich die Frage, ob und wie Recht und Willkür zu unterscheiden sind. Wäre der Geltungsanspruch alleiniges Merkmal der Rechtsnorm, müßte jeder Befehl auch eines Verbrechers, der Gehorsam gegenüber seinen Anweisungen fordert, einem staatlichen Gesetz gleichgestellt werden. Stammler entnimmt auch den Unterschied von Recht und Willkür der Erfahrung: „Willkürliche Gewalt liegt vor, wenn ein Befehl erlassen wird, den

${ }^{1}$ Rudolf Stammler, Recht und Willkür (1895), in: ders., Rechtsphilosophische Abhandlungen und Vorträge, Bd. 1 (Charlottenburg 1925) 85-118.

2 Eine erste Annäherung habe ich in dem folgenden Beitrag versucht: Rudolf Stammlers Abhandlung "Recht und Willkür ${ }^{\text {" }}$ und ihre Konsequenzen für den Rechtsbegriff, in: Perspektiven der Philosophie 23 (1997) 181-192 mit weiteren Nachweisen. 
der Betreffende selbst gar nicht - dem formalen Sinne nach - als objektiv bindende Regelung menschlicher Verhältnisse erachtet; der vielmehr, seiner eigenen Meinung nach, nur die formale Bedeutung einer Befriedigung subjektiver Gelüste und Wünsche des Gewalthabers durch ledigliche Bindung anderer hat, auf den er zurückkommen wird, wenn er wollen wird; der aber jetzt, bei seinem Erlasse, nichts von einer Bindung des Befehlenden an seinen Befehl in sich trägt. Das Gegenstück ist dann gegeben, wenn der Gebietende an die von ihm erlassene Regel selbst auch gebunden sein will. Es müssen beide an das Gebot gebunden sein. Wenn der $\mathrm{Ge}-$ bietende nicht gebunden ist, so ist es rohe Willkürmacht ...". Daraus ergibt sich die Definition: „Recht ist ihrem Sinne nach unverletzbar geltende $Z$ wangsregelung menschlichen Zusammenlebens. ${ }^{3}$ Dieser Rechtsbegriff erlaubt zunächst die Annahme, daß Recht auch aus Rechtsbruch, z. B. durch eine Revolution, hervorgehen kann. Wenn andererseits das Außerkrafttreten des gebrochenen Rechts, das seinem immanenten Sinne nach ja weitergetten möchte, kaum erklärt werden kann, so ist das nur eine scheinbare Schwäche dieser Begriffsbildung. Sie geht von der Erfahrung aus und läßt sich von der Erfahrung auch belehren, daß Recht mißachtet wird: „Das Recht kann gebrochen werden, aber es will es nicht; es stirbt, doch es ergibt sich nicht. Und solange es lebt, so eignet ihm auch der Anspruch, der sein Wesen ausmacht und ihm das selbständige Dasein erst verschafft: - der Anspruch der Unverletzbarkeit." ${ }^{4}$

Diese Orientierung am empirischen Befund, alle diese "Wahrnehmungen, daß auf ganz verschiedenartigem Wege und namentlich auch außerhalb der von dem Rechte selbst für neue Rechtsbildung aufgestellten Regeln Rechtssätze in das Leben treten können, (führen) letztiich auf die allgemeine Frage zurück: Woran erkennt man überhaupt, ob etwas Recht ist? - Hierfür muß es doch ein allgemeingültiges Merkmal geben. " 5 Diese Frage führt nun zu der weiteren nach "der originären Entstehung von Recht" und damit, ohne daß dies der Autor besonders hervorhebt, zu den historisch ursprünglichen Konstellationen, aus denen Recht hervorgeht. Es sind „zwei Möglichkeiten zu unterscheiden: eine solche der Einigung unter den Recht Setzenden und nun zugleich rechtlich Gebundenen, oder aber eine einseitige Setzung durch einen Machthaber gegen den Willen des Unterworfenen"6. Der Einigung unter den Beteiligten, d.h. dem Vertrag, kommt deshalb eine rechtserzeugende Wirkung zu, „weil ja im Begriffe der Vereinbarung das Moment sich notwendig erfüllt, welches die rechtliche Satzung von willkürlicher Maßnahme allgemeingültig trennt: das Moment der Unverletzbarkeit während ihrer Geltung "7. Ohne es ausdrücklich zu sagen, hat sich Stammler mit diesen Beobachtungen aus den Rahmenbedingungen einer staatlich organisierten Rechtsordnung hinausbewegt. Liegt der Sinn von Recht darin, Willkür auszuschließen,

3 Stammler, (wie Anm. 1) 97 u. 98.

4 Stammler, (wie Anm. 1) 98.

5 Stammler, (wie Anm. 1) 104.

6 Stammler, (wie Anm. 1) 105.

7 Stammler, (wie Anm. 1) 105. 
dann muß der Rechtsgedanke schon in elementaren sozialen Beziehungen vor der Herausbildung staatlicher Verhältnisse begründet sein.

Stammlers Rechtsbegriff läßt sich an bestimmten Erscheinungen der Rechtsgeschichte kontrollieren und erweist sich dabei als ein Vorbegriff von positivem Recht, der dessen fundamentalste Normen hervorragend zu erklären vermag. Damit komme ich zu den versprochenen rechtsgeschichtlichen Hypothesen. Weil Willkür mit dem Rechtsgedanken nicht vereinbar ist, deshalb gibt es soziale Sachverhalte, die man als ein rechtliches Minimum menschlicher Vergesellschaftung ansprechen kann. Es handelt sich erstens um das Vertrauen in die Beständigkeit vertraglicher Vereinbarungen und zweitens um das Genugtuungsverlangen des Geschädigten nach einer Verletzung seiner Güter. Zwischenmenschliche Beziehungen, in denen das Verlangen nach einer Sühne, also nach einem irgendwie gearteten Ausgleich für geschehene Verletzungen, nicht anerkannt ist und die auch ein Vertrauen in die Bindungswirkung von Vereinbarungen nicht kennen, können wir uns als rechtlich geordnete nicht vorstellen. Sie wären eine pervertierte Form menschlichen Zusammenlebens, weil Einbußen an Leib, Leben und Gütern stumpf hingenommen werden müßten und das Wort eines anderen keinen Glauben verdiente. Menschliches Zusammenleben ließe sich dauerhaft so nicht organisieren. Die Menschen einer solchen Gesellschaft würden leben ohne Recht. Sie wären dem willkürlichen Verhalten anderer ausgeliefert. Wenn zum Rechtsbegriff Normativität gehört, wie kaum jemand bestreiten würde, dann muß sich die Norm im Sinne der von Stammler betonten Selbstbindung auch ernst nehmen und damit zugleich normungebundenes, also willkürliches, Verhalten als Unrecht disqualifizieren.

\section{III.}

Damit komme ich zu Rückfragen, welche die Rechtsgeschichte des 20. Jahrhunderts provoziert. Stammler hilft uns auch insofern noch ein Stück weiter. Er hat, als er seine Abhandlung niederschrieb, noch nichts von den Horrorszenarien des 20. Jahrhunderts gewußt, von den rassischen und politischen Verfolgungen im Dritten Reich, von den Säuberungen in den Sowjetstaaten, von Rechtsbrüchen und Terrorakten, die Staaten selbst organisieren. Aber er fand in der europäischen Geschichte den absoluten Herrscher und in der staatswissenschaftlichen Tradition die Figur des Despoten vor und stellte sich die Frage, ob wir es in diesen Fällen noch mit Formen "rechtlicher Verfassung " zu tun haben. Stammler unterscheidet beide sehr sorgfältig. Absolutismus nennt er „eine rechtliche Verfassung, nach der die Rechtsverhältnisse der Untertanen nach im voraus bestimmten Sätzen geregelt sind, deren Abänderung und Ergänzung durch den Willen eines einzigen Menschen geschieht ${ }^{\text {. }}$. Dagegen würde "Despotismus ... in seiner vollendeten Art eine Rechtsordnung heißen, die nur aus einem einzigen, im voraus allgemein aufgestellten Paragraphen bestände: Die rechtlichen Beziehungen unter den Rechts- 
unterworfenen werden lediglich nach der konkreten Entscheidung des Herrschers im einzelnen Falle beurteilt und durchgeführt. ${ }^{\text {8 }}$ Stammler nennt auch dies noch eine ${ }_{n}$ rechtliche Verfassung “, weil der ${ }_{n}$ angeführte Rechtssatz ... seinem Sinne nach als eine während ihres Bestehens unverletzbare Norm (gilt)". Es sei dies „nicht bloß eine von Fall zu Fall zufällig sich ereignende Gewalt und Macht; denn sonst würde die jeweilige größere Körperkraft oder der konkrete sich gerade bewährende psychische Einfluß des Despoten allein zur Unterlage genommen. Dem Sinne des despotisch regierten Gemeinwesens aber entspricht es, daß die einzelnen Akte auf jenen angeführten Satz zurückgehen; und die Ausführung der $\mathrm{Be}$ fehle durch Organe und Diener des Despoten geschieht daher an sich von Rechts wegen." Man könne daher „im ruhigen Zustande der Despotie“ unterscheiden, „ob der Despot als Herrscher eine durch nichts im voraus sachlich beschränkte Anordnung als Rechtsbefehl erläßt oder ob er als Mensch sich vergeht, jemanden ermordet, ein Mädchen notzüchtet"9. Stammler scheint sich in der Tat die Ausübung physischer Gewalt oder vergleichbarer Mittel als Grenze vorgestellt zu haben, an welcher das Recht endet und die Willkür beginnt. Am weitesten wagt er sich mit der folgenden Formulierung vor: „Wenn man sich freilich in der Hypothese einmal einen Zustand denken wollte, in welchem jemand nur mit Körperkraft oder eigentümlich psychischem Einflusse - oder sagen wir phantastisch: mit ihm allein bekannter Maschinerie - vielleicht hundert Menschen als Sachen zu beliebigem Gebrauche nach momentaner Laune behandelte, so wäre auch der letzte Rest einer rechtlichen Verfassung abgestreift; und nichts als ein Gemisch von Willkürakten zurückgeblieben, die sozial in keiner Einheit zusammenflössen." ${ }^{10}$

Mit diesen Ausiassungen verspielt Stammler, was er zuvor durch seine sorgfältigen Überlegungen zum Rechtsbegriff gewonnen hat. Sein Versuch, die äußersten Ränder des Rechtsbegriffs abzugehen, führt in die Irre, weil er jetzt plötzlich sein eigenes Kriterium für Recht, die Selbstbindung des Rechtsetzenden, vergißt. Der Einsatz von Körperkraft oder eines "eigentümlich psychischen Einflusses" oder die Benutzung einer dem Despoten allein bekannten Maschinerie könnte ebensogut zur Durchsetzung von Rechtssätzen wie auch willkürlich geschehen. Ebensowenig überzeugt der Vorschlag, man solle danach unterscheiden, ob der Despot „als Herrscher “ oder „als Mensch“ handele. Eben dies würde man im Zweifelsfalle ja gerne wissen. Der Verdacht drängt sich auf, Stammler könnte hier deshalb auf Abwege geraten sein, weil er vor der Konsequenz seines Rechtsbegriffs, Staatsgewalt - und sei es diktatorische - unter Umständen für unrechtmäßig erklären zu müssen, zurückschreckte.

In Wirklichkeit kommt man mit Stammlers Rechtsbegriff den Problemen der Despotie und damit auch den historischen Ereignissen des 20. Jahrhunderts viel näher, als dies Stammler selbst wahrhaben wollte. Recht hat er insofern, als sein Rechtsbegriff gegen beliebige Änderungen des Rechts in der Tat keine Sicherheit

8 Stammler, (wie Anm. 1) 111.

9 Stammler, (wie Anm. 1) 111.

10 Stammler, (wie Anm. 1) 113. 
bietet $^{11}$. Aber wenn der Rechtscharakter einer Norm erfordert, daß sich die normsetzende Autorität selbst an diese Norm binden will, dann ist es möglich, innerhalb der staatlichen Hoheitsakte zwischen Normsetzung mit Selbstbindungswillen und Befehlen willkürlicher Art zu unterscheiden. Nicht nur dann, wenn der Despot selbst mordet oder Mädchen notzüchtigt, handelt er willkürlich, sondern auch, wenn er Gebote erläßt, an die er sich je nach persönlicher Lust oder politischer Neigung halten wird oder auch nicht. Stammler kann mit seiner Feststellung, in der Despotie würden eben "die rechtlichen Beziehungen unter den Rechtsunterworfenen ... lediglich nach der konkreten Entscheidung des Herr-

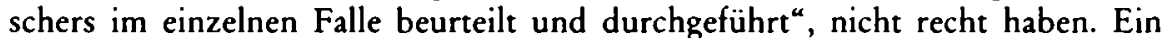
solcher Satz enthält den Versuch, Willkür als Recht zu institutionalisieren, und widerspricht sich damit selbst. Bald nachdem Stammler diese verunglückte Paradoxie zu Papier gebracht hatte, entstanden in der geschichtlichen Wirklichkeit Verfassungssysteme, die genau dieses versuchten: das Recht auf eine jeweilige, konkrete Entscheidungssituation zu gründen, sei es auf den Willen des Führers, sei es auf die Diktatur des Proletariats zum Zwecke des Klassenkampfes. Führerprinzip und klassenkämpferische Diktatur binden sich schon ex definitione nicht an die eigenen Normen, weil ihnen an der Normativität ihrer Gebote nichts liegt, sondern Führung und Kampf die entscheidende Rolle zu spielen haben. Damit aber nehmen diese politischen Systeme eine beliebige Entscheidungsfreiheit für sich in Anspruch, die sich mit dem Rechtsbegriff nicht verträgt. Nicht zufällig sind sowohl im Dritten Reich wie auch in der DDR Rechtsnormen, die eigentlich "galten ", vielfach nicht beachtet worden. Die Juristen dieser Staaten haben das sehr wohl gespürt. Bekannt ist das Dilemma der nationalsozialistischen Jurisprudenz, den Führerwillen doch noch normativ durch Bindung an "Blut“ und "Volksempfinden" einfangen zu wollen ${ }^{12}$. In der DDR kam eine ähnliche Funktion dem seit den sechziger Jahren erörterten Prinzip der "sozialistischen Gesetzlichkeit ${ }^{\text { }} \mathrm{zu}^{13}$. Sicher gibt es auch insofern große Unterschiede zwischen den beiden deutschen Diktaturen. Aber daß das politische Antriebssystem in beiden Staaten weniger nach normativen Vorgaben als nach politischer Zweckmäßigkeit funktionieren sollte, wird man kaum bestreiten können. Nur am Rande möchte ich zu bedenken geben, daß die Einsichten, die Rudolf Stammlers Unterscheidung von Recht und Willkür nahelegen, vielleicht deshalb in der rechtswissenschaftlichen Diskussion des 20. Jahrhunderts untergepflügt wurden, weil Carl Schmitt die Rechtsfremdheit von Führertum und Kampf unter dem Banner der „Dezision" ins Positive wendete ${ }^{14}$.

Es ist leicht zu erkennen, daß sich auch mit Stammlers Rechtsbegriff nicht alle Fragen zur Rechtsgeschichte des 20. Jahrhunderts beantworten lassen. Die Nürn-

11 Stammler, (wie Anm. 1) 98.

12 Diemut Majer, Grundlagen des nationalsozialistischen Rechtssystems (Stuttgart u.a. 1987).

13 Georg Brunner, Einführung in das Recht der DDR (München 21979).

14 Hasso Hofmann, Legitimität gegen Legalität. Der Weg der politischen Philosophie Carl Schmitts (Berlin 21992) $62 \mathrm{ff} ., 68 \mathrm{f}$. 
berger Rassegesetze sind zweifellos mit dem Selbstbindungswillen des damaligen Gesetzgebers erlassen und auch exekutiert worden. Das Problem, ob und inwiefern es auch willkürliche Normsetzung geben kann oder ob dies eine in sich widersprüchliche Begriffsbildung wäre, kann an dieser Stelle nicht gelöst werden. Sicher scheint mir indessen, daß Stammlers Rechtsbegriff mehr leistet als der moderne Rechtspositivismus, der den Rechtsbegriff schon erfüllt sieht, wenn Normen „den internen Verfassungsprinzipien der jeweiligen Rechtsordnung entsprechen ", wie es Norbert Hoerster ausdrückt ${ }^{15}$. Dieser Satz kann nur insofern richtig sein, als eine Verfassung nicht willkürliches Verhalten des oder der Machthaber zum Prinzip erhebt.

Man mag sich fragen, welches denn der Ertrag der bisherigen Überlegungen für das Problem der Rechtsbegründung sein könnte. Wenn der Rechtsbegriff definitorisch eingegrenzt wird, dann gehen natürlich Begründungsversuche ins Leere, die über den Raum des Rechts hinaus politisches Handeln überhaupt zu begründen versuchen. Diese Disproportionalität zwischen Rechtsbegriff und Legitimierungsbedarf festzustellen, scheint mir aber nicht unwichtig. Zum einen ist die Tatsache, daß sich das Legitimationsbedürfnis über die Phänomene des Rechts hinaus erstreckt und per se auch nicht erkennbar ist, wo es enden sollte, für die Legitimationsproblematik an sich von erheblichem Interesse. Eine Entdeckung ist dies nun keineswegs. Wir erinnern uns, daß Max Weber die Legitimitätsfrage nicht an das Recht, sondern an die „Herrschaft“ geknüpft hat. Er ging von „dem sehr allgemeinen 'latbestand des Bedürfnisses jeder Macht, ja jeder Lebenschance überhaupt, nach Selbstrechtfertigung " aus ${ }^{16}$. So gesehen, spiegelt der Fragenkreis der Rechtsbegründung nur einen Teil der Legitimationsproblematik wider. Und es drängt sich die Vermutung auf, daß dort, wo nicht rechtliches, sondern willkürliches Handeln begründet werden soll, ein ganz außergewöhnlicher Argumentationsbedarf gegeben ist - man denke nur an die quasireligiöse Gläubigkeit, mit der im Dritten Reich das Führerprinzip gepriesen wurde, oder an die intellektuellen Energien, die sich um die Begründung der klassenkämpferischen Diktatur bemühten.

Der Befund, daß das Legitimierungsbedürfnis über das Recht hinausreicht, zicht andererseits eine Entlastung der Rechtsbegründungsfrage nach sich. Dieser Aspekt ist für meine Fragen an die Geschichte von erheblich größerer Bedeutung. Die historisch verifizierbaren Modelle der Rechtsbegründung müssen das willkürliche Handeln eines Despoten nicht abdecken. Speziell die historische Last des 20. Jahrhunderts muß sich nicht aufladen, wer rückblickend danach fragt, wo die verschiedenen Ansätze der Rechtsbegründung eventuell konvergieren. Denn an dieser Frage nach den Gemeinsamkeiten legitimierenden Rechtsdenkens über Epochen hinweg wird die rechtsgeschichtliche Forschung und überhaupt die

15 Norbert Hoerster, Verteidigung des Rechtspositivismus (Würzburger Vorträge zur Rechtsphilosophie, Rechtstheorie und Rechtssoziologie 11) (Frankfurt am Main 1989) 11. 16 Max Weber, Wirtschaft und Gesellschaft, hrsg. v. Johannes Winckelmann (Tübingen 51972) 549. 
Rechtswissenschaft nicht vorbeikommen. Max Webers Soziologie der Herrschaft bietet ein methodisches Vorbild und inhaltliche Hinweise, um die Fülle der historischen Einzelbefunde mit Hilfe einer typenbildenden Begrifflichkeit wenigstens teilweise zu verstehen. So kommt in der europäischen Geschichte sicher sakralen Autoritäten, dem Willen des Volkes und dem rechtmäßigen Herkommen eine hervorragende Bedeutung für die Rechtfertigung von Recht zu. Daher konnten Gebot, Vertrag und Sitte als nUrformen der Begründung von Rechtsverbindlichkeit " bezeichnet werden ${ }^{17}$. Aus der Beobachtung der scheinbar in sich ganz widersprüchlichen Rechtsbildung des Mittelalters ergibt sich allerdings auch die Frage, ob die Rechtsordnung letztlich nicht als ein Gefüge von Konsensbeziehungen zu begreifen ist ${ }^{18}$, das durch sakrale Elemente und rationale Begründungen der politischen Theorie dem individuellen Willen entzogen und auf diese Weise immer wieder gefestigt werden muß.

17 Hasso Hofmann, Gebot, Vertrag und Sitte. Die Urformen der Begründung von Rechtsverbindlichkeit (Würzburger Vorträge zur Rechtsphilosophie, Rechtstheorie und Rechtssoziologie 17) (Baden-Baden 1993).

18 Dietmar Willoweit, Vom alten guten Recht. Normensuche zwischen Erfahrungswissen und Ursprungslegenden, in: Jahrbuch des Historischen Kollegs 1997 (München 1998) 23ff., $39 \mathrm{ff}$. 